\title{
Re: Minimally Invasive, Laparoscopic, and Robotic-Assisted Techniques versus Open Techniques for Kidney Transplant Recipients: A Systematic Review
}

\author{
Wagenaar S1, Nederhoed $\mathrm{JH}^{2}$, Hoksbergen $\mathrm{AW}^{2}$, Bonjer $\mathrm{HJ}^{2}$, Wisselink $\mathrm{W}^{2}$, van Ramshorst $\mathrm{GH}^{3}$ \\ ${ }^{1}$ VU University Medical Center, Department of Surgery, Amsterdam, the Netherlands; Meander Medisch Centrum, Clinic of Urology, Amersfoort, \\ the Netherlands \\ ${ }^{2}$ VU University Medical Center, Department of Surgery, Amsterdam, the Netherlands \\ ${ }^{3} \mathrm{VU}$ University Medical Center, Department of Surgery, Amsterdam, the Netherlands; the Dutch Cancer Institute/Antoni van Leeuwenhoek \\ Hospital, Clinic of Surgery, Amsterdam, the Netherlands
}

Eur Urol 2017 pii: S0302-2838:30109-4. doi: 10.1016/j.eururo.2017.02.020.

\section{EDITORIAL COMMENT}

In recent years, application of minimally invasive techniques (MIT) mainly open minimally invasive, laparoscopic and robotic surgery into kidney transplantation has become more popular in kidney donors than in kidney recipients. In this paper, the authors have systematically reviewed the limited data of MIT in kidney transplant recipients compared to open techniques for the first time. The results have shown no difference in terms of graft and patient survival. For open techniques, the Gibson incision has proved to show better results for incisional hernia, abdominal wall relaxation and cosmesis compared to hockey-stick incision. On the other side, as expected, less surgical site infections, incisional hernia, improved postoperative recovery with better cosmetic results have been achieved with MIT compared to open techniques. Unsurprisingly, the trade-off was for prolonged cold ischemia time, warm ischemia time and total operation time. Still the laparoscopic and robotic surgeries are not the standard of care in the management of kidney recipients; there is an undeniable leaning towards MIT especially in the robotic surgery arm.

Yarkın Kamil Yakupoğlu, MD 